\title{
Acoustic Mode Coupling by Nonlinear Internal Wave Packets in a Shelfbreak Front Area
}

\author{
Timothy F. Duda
}

\begin{abstract}
A computational case study of coupled-mode $400-\mathrm{Hz}$ acoustic propagation over the distance $27 \mathrm{~km}$ on the continental shelf is presented. The mode coupling reported here is caused by lateral gradients of sound-speed within packets of nonlinear internal waves, often referred to as solitary wave packets. In a waveguide having unequal attenuation of modes, directional exchange of energy between low- and high-loss modes, via mode coupling, can become time dependent by the movement of waves and can cause temporally variable loss of acoustic energy into the bottom. Here, that bottom interaction effect is shown to be sensitive to stratification conditions, which determine waveguide properties and, in turn, determine modal attenuation coefficients. In particular, timedependent energy loss due to the presence of moving internal wave packets is compared for waveguides with and without a frontal feature similar to that found at the shelfbreak south of New England. The mean and variability of acoustic energy level $27 \mathrm{~km}$ distant from a source are shown to be altered in a first order way by the presence of the frontal feature. The effects of the front are also shown to be functions of source depth.
\end{abstract}

Index Terms-Continental shelf, internal waves, mode coupling, shallow water, shelfbreak front, solitary waves, sound propagation.

\section{INTRODUCTION}

$\mathbf{T}$ HE exchange of energy between propagating acoustic normal modes caused by the sound-speed heterogeneities present in nonlinear internal waves has been shown to have important consequences for acoustic signals at a few hundred hertz propagating in the shallow-water waveguide [1]-[4]. Propagation characterized by such an exchange between modes is generally called coupled mode propagation. High-amplitude nonlinear internal gravity waves have highly sloped isopycnals (sound speed isotachs), which correspond to high horizontal gradients of sound speed, which in turn cause mode coupling. Thus, the waves have first order effects on acoustic fields. Mathematically, the rate (with respect to range) of mode coupling can be approximated by an expression containing a depth integral of a triple product, the factors being each of the two mode shapes and the range derivative of a characteristic wavenumber [5]. In the case of constant depth, the derivative can be rewritten to be proportional to the range derivative of sound speed, which in turn can be shown to be proportional to isotach slope for idealized stratification structures [1].

Manuscript received March 25, 2003; revised September 5, 2003. This work was supported by the Office of Naval Research Grants N00014-99-1-2074 and N00014-01-1-0772. This is Woods Hole Oceanographic Institution Contribution 10926.

The author is with the Applied Ocean Physics and Engineering Department, MS 11, Woods Hole Oceanographic Institution, Woods Hole, MA 02543 USA (e-mail: tduda@whoi.edu).

Digital Object Identifier 10.1109/JOE.2003.822975
Although mode coupling by moving wave packets will probably always induce greater temporal acoustic field variability than would exist in the absence of the packet [2], [3], the effect on temporally averaged signal strength at ranges greater than a few kilometers down range from a packet may be either amplification or attenuation, depending on signal and waveguide characteristics. This net (temporally averaged) gain effect of packet coupling depends strongly on four factors.

1) Modal composition of the acoustic field encountering the packet, which is determined by the source depth, the sound-speed profile in the area between the source and the receiver, the bottom composition, and the distance between the source and the packet.

2) Distance between the packet and the receiver.

3) Acoustic waveguide character at the site of the waves.

4) Acoustic waveguide character between the waves and the receiver.

This computational study examines packet-coupling effects on signal strength in two environments that are characteristic and representative of conditions in the summer in many temperate shelf regions. Thus, two cases of factor 3 are considered. The two environments also correspond to different cases of factor 1 because the profiles at the source differ. They also correspond to different cases of factor 4 . Source-to-receiver distance is held constant. In each of the two cases, a complete range of packet position is considered, giving a complete range of packet-toreceiver distance (factor 2 ) and of source-to-packet distance (a contributor to factor 1). Three source depths (a contributor to factor 1) are considered for each environment, giving six cases overall.

One environment has a strong pycnocline in the upper portion of the water column; the other has the same pycnocline plus an additional pycnocline close to the bottom, where salty warm offshore water underlies water that has been freshened by river runoff. The second environment is intended to correspond to conditions observed on occasion during the Shelfbreak PRIMER experiment [6] and the Coastal Mixing and Optics Experiment [7]-[9] (Fig. 1). The interface between the salty warm water and the water above in that area has been called the shelf/slope water front and the shelfbreak front. Mode-one nonlinear internal waves take the form of depression waves in both situations (with and without the front) because the upper layer is thinner than the lower layer. This study is an extension of an earlier computational study [10]; the earlier results are a subset of what is presented here.

The paper is organized as follows. Section II describes the two environments and the computational setup. Section III shows the 
(a) $\mathrm{T}\left({ }^{\circ} \mathrm{C}\right)$

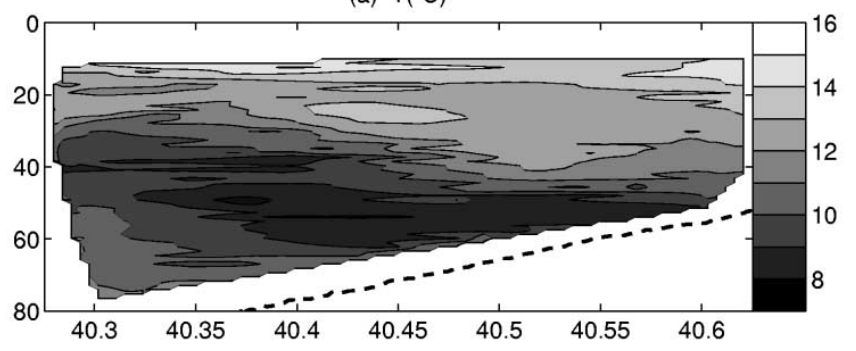

(b) $\mathrm{S}$ (psu)

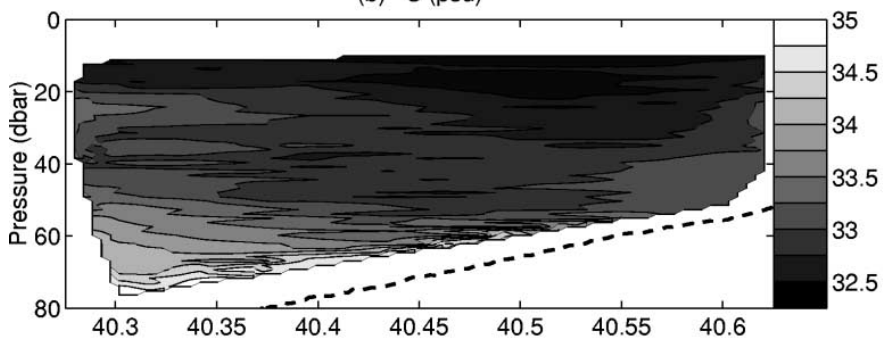

(c) $\mathrm{C}(\mathrm{m} / \mathrm{s})$

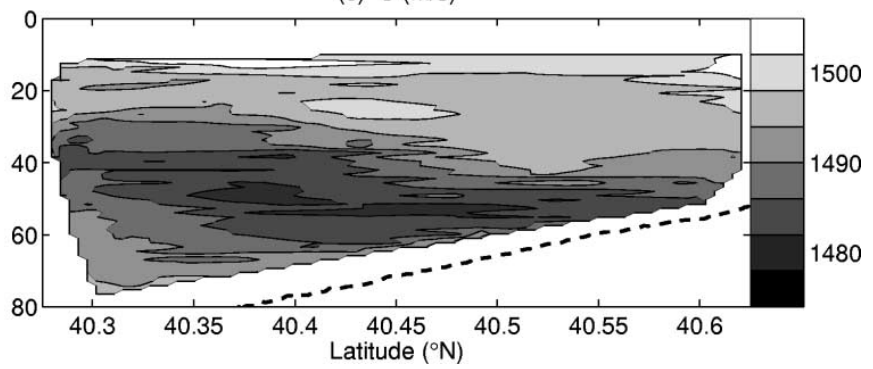

Fig. 1. Measurements along a roughly meridional section of (a) temperature, (b) salinity, and (c) sound speed taken during the 1997 Coastal Mixing and Optics study [8] are shown. Over much of the section there are three layers: a warm layer with high sound speed at the surface, a cooler layer with low sound speed just below that, and a warm salty layer with high sound speed at the bottom.

results of the simulated propagation for the two environments and Section IV discusses the findings.

\section{COMPUTATIONAL PARAMETERS}

The problem is addressed using monochromatic 400 $\mathrm{Hz}$ propagation simulations performed in two-dimensional (2-D)slices. A modified version of the RAM parabolic equation code is used, which utilizes methods published by Collins [11]. The range step is $1 \mathrm{~m}$, the vertical grid spacing is 0.25 $\mathrm{m}, c_{0}=1488 \mathrm{~m} / \mathrm{s}$, and the Pade expansion parameter is 2 . We have modified the code so that sea-bed environmental parameters are constant with range in a coordinate system tied to the bathymetry until they are updated with a new set of parameters supplied to the marching algorithm, eliminating the need to input bottom parameters into memory at every range step in a simulation over a sloping bottom.

The two environments have the same bathymetry and bottom properties; only the background stratification differs. The acoustic source site has a water depth of $130 \mathrm{~m}$. Between the source and receiver sites, $27 \mathrm{~km}$ from the source, the sea floor is composed of six uniformly sloping sections. The depth remains $130 \mathrm{~m}$ for the first $3 \mathrm{~km}$, decreases to $120 \mathrm{~m}$ at $6-\mathrm{km}$ range, decreases to $100 \mathrm{~m}$ at $9 \mathrm{~km}$, decreases to $90 \mathrm{~m}$ at $15 \mathrm{~km}$,
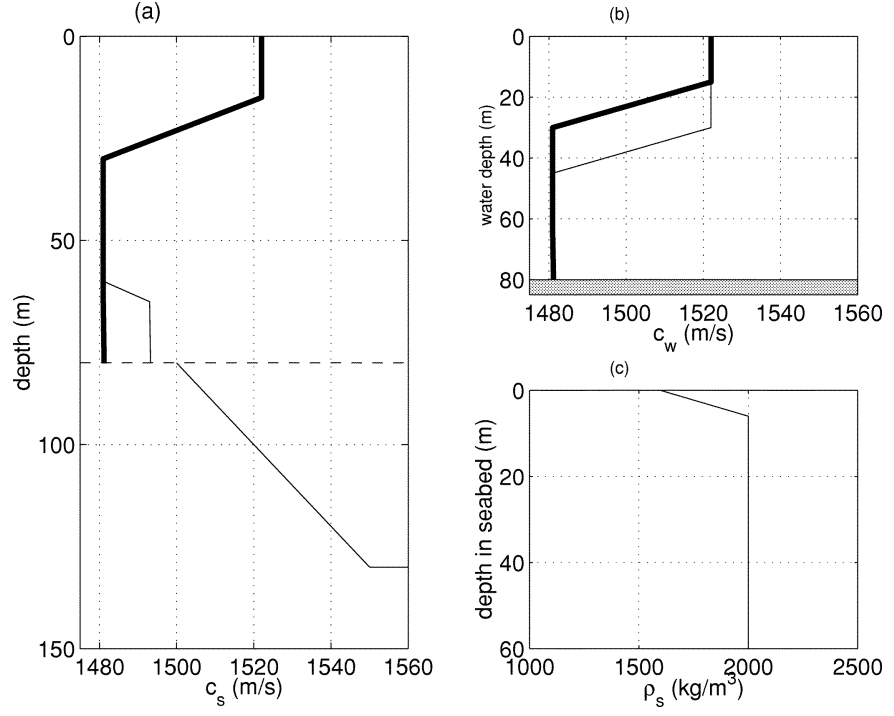

Fig. 2. (a) Background stratifications (converted to sound speed) with and without the warm deep (high sound speed) layer (the frontal structure) are shown, along with the sound speed in the sea bed. (b) The effect of a 15-m internal wave of depression is illustrated. The background profile (thick line) is perturbed at the center of the wave to the displaced profile shown. (c) The density in the sea bed is shown.

TABLE I

Six Computational Scenarios (CASES A Through F) ARE Listed

\begin{tabular}{ccc}
\hline Source depth $(\mathrm{m})$ & Without deep layer (front) & With deep layer (front) \\
\hline 20 & $\mathrm{~A}$ & $\mathrm{~B}$ \\
50 & $\mathrm{C}$ & $\mathrm{D}$ \\
100 & $\mathrm{E}$ & $\mathrm{F}$ \\
\hline
\end{tabular}

decreases to $80 \mathrm{~m}$ at $18 \mathrm{~km}$, and finally decreases to $70 \mathrm{~m}$ at $27 \mathrm{~km}$. One domain has a downward-refracting thermocline structure typical of summer conditions and the other is identical except for the addition of the deep layer of warm salty (high sound speed) water intruding onto the shelf (i.e., the addition of the front).

The background stratification in both environments, shown in Fig. 2(a), has a 15-m-deep upper layer of sound speed $c_{w}=$ $1522 \mathrm{~m} / \mathrm{s}$, a layer of $c_{w}=1481 \mathrm{~m} / \mathrm{s}$ extending from 30 to 65 $\mathrm{m}$ depth, and a 15-m-deep transition layer connecting the two [Fig. 2(a)]. Below a 65-m depth, the sound speed increases linearly with depth from $1481 \mathrm{~m} / \mathrm{s}$ at $65-\mathrm{m}$ depth at a rate of $1 \mathrm{~m} / \mathrm{s}$ per $65 \mathrm{~m}$. The front-type environment [Fig. 2(a)] is similar, but adds a step-like linear $12-\mathrm{m} / \mathrm{s}$ increase of $c_{w}$ between 60 - and 65-m depth, with the same gradient existing at depths greater than $65 \mathrm{~m}$, so that a sound channel exists between $15-$ and $65-\mathrm{m}$ depth. The sound speed in the sea bed is also shown in Fig. 2(a) and gives sound speed a duct-like character in the water and the upper $50 \mathrm{~m}$ of sea bed. However, the high density in the sea bed [Fig. 2(c)] and attenuation in the sea bed of 0.1-dB/wavelength keeps the sound effectively trapped in the water. The main conclusions to be drawn by comparing the six cases studied here (Table I) are not strongly sensitive to sound speed in the bottom, although details of each case depend on this choice. The model is intended to mimic the behavior of mud, rather than sand or gravel (see, for example, [12]). 

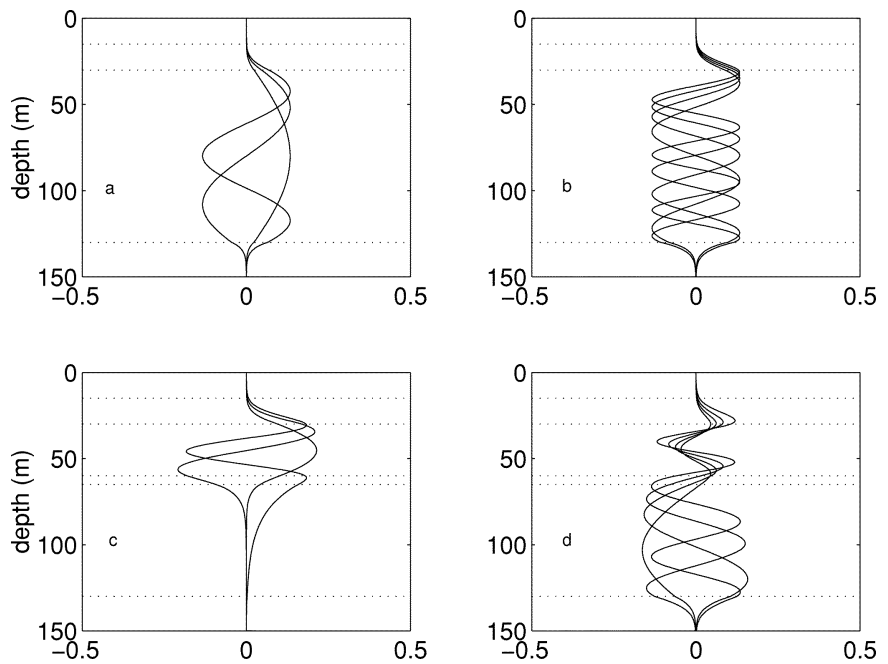

Fig. 3. 400-Hz acoustic mode shapes at the source position are shown for the two environments. At the top, (a) and (b) show modes 1-3 and 4-7, respectively, for the environment without the deep warm layer. At the bottom, (c) and (d) show the same modes for the environment with the deep warm layer. The dotted lines indicate the boundaries of the various sound-speed layers (15-, 30-, 60-, and 65-m depth) and the bottom. Note that modes 4-6 have a higher magnitude in the bottom $60 \mathrm{~m}$ of the water column than at shallower depths in panel (d), i.e., they are bottom enhanced, but this is not true in (b).

(a)
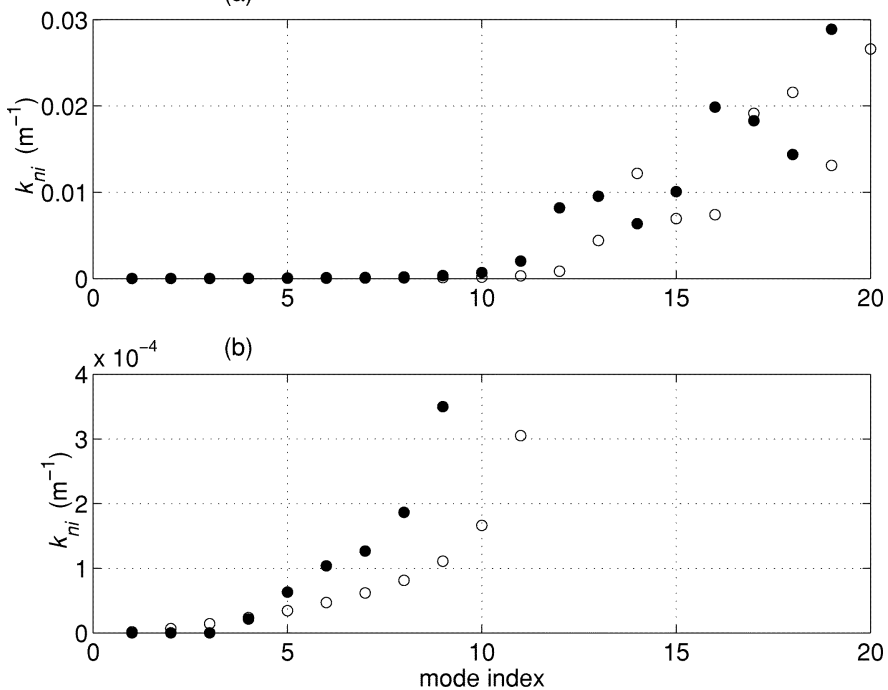

Fig. 4. Mode attenuation coefficients $k_{n i}$ computed at the 130-m depth source position are shown for the first 20 modes. The same points are plotted in the two frames, which have different y-axis scales. The open circles show values for the environment without the warm lower layer. The filled circles show values for the environment with the warm layer (i.e., with the front).

Fig. 3 shows the acoustic mode shapes in each of the two environments at the source location. Note the changes that the presence of the lower layer (the front) impart on modes 1-3, which do not interact with the bottom when the layer is present. Note also that modes 4-7 have higher amplitudes in the lower layer than in the intermediate layer (i.e., they are bottom enhanced) for the case with the layer, but not for the other case. The imaginary components of the complex modal eigenvalues $\left(k_{n i}\right.$, where the modal eigenvalues are $k_{n}=k_{n r}+i k_{n i}$ ) are plotted in Fig. 4 for both environments, again at the source site of 130-m depth. These correspond to modal attenuation coefficients with units of inverse meters and are inverses of e-folding distances of modal amplitude under adiabatic mode-propagation conditions.

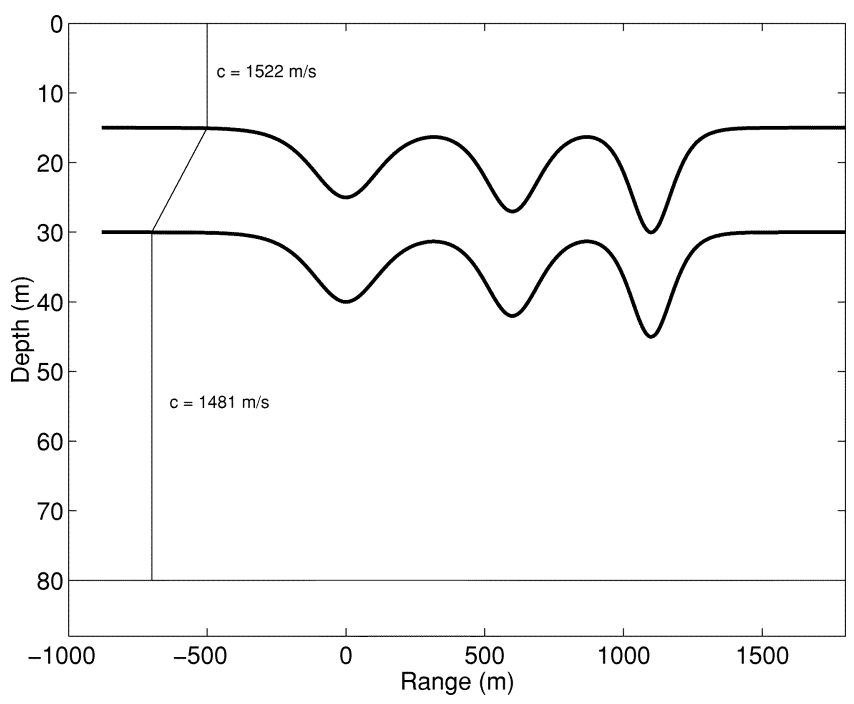

Fig. 5. Packet of three $\operatorname{sech}^{2}$-shaped upper-interface waves of depression used in the simulations is shown.

Modes with index higher than 10 are always highly attenuated. Without the front (warm layer), modes 1-10 show gradually increasing attenuation. With the front (warm layer), modes 1-3 are essentially not attenuated and modes 5-13 show more attenuation than in the no-front case.

Three source depths of 20,50 and $100 \mathrm{~m}$ are considered, giving a total of six case studies (Table I). The 20 -m source is always in the upper transition layer and the $50-\mathrm{m}$ source is always in the duct. In case $\mathrm{C}$, the duct is strongly bottom interacting and in case $\mathrm{D}$ it is not. The $100-\mathrm{m}$ source is in the bottom interacting duct in case $\mathrm{E}$, but is below the duct in case $\mathrm{F}$.

In each case study, an internal solitary wave packet of approximately $2-\mathrm{km}$ length rides on the background stratification. The packet is composed of three waves and moves with unaltered geometry from offshore of the source site (in deeper water than the receiver) to inshore of the receiver site, in a manner comparable to the simulations of Duda and Preisig (1999), although that study used a simpler domain of constant depth. The packet of $\operatorname{sech}^{2}$-shaped waves, similar to those of Duda and Preisig (1999), is shown in Fig. 5. The amplitudes are 10, 12, and $15 \mathrm{~m}$ and the horizontal length scales are 160,130 , and $100 \mathrm{~m}$. As in the previous work, the amplitudes and length scales of the waves are not constrained to follow solutions of the Korteweg-deVries equation because the horizontal scale of the waves in the 2-D acoustic slice is stretched if the waves do not propagate in the same direction as the sound. The movement of the packet is simulated by running many independent simulations with the packet at differing ranges, with a packet-range increment of 25 $\mathrm{m}$. When the wave packets are outside of the acoustic propagation domain (not between the source and receiver), there is no range dependence to the sound-speed profile and the acoustic propagation exhibits no mode coupling (i.e., is adiabatic). These situations form control runs for each of the six cases.

\section{RESULTS}

\section{A. Effect of the Deep Layer}

We first consider the propagation characteristics in the absence of wave packets. Fig. 6(a) shows the acoustic field inten- 
(a) Case A, no packet

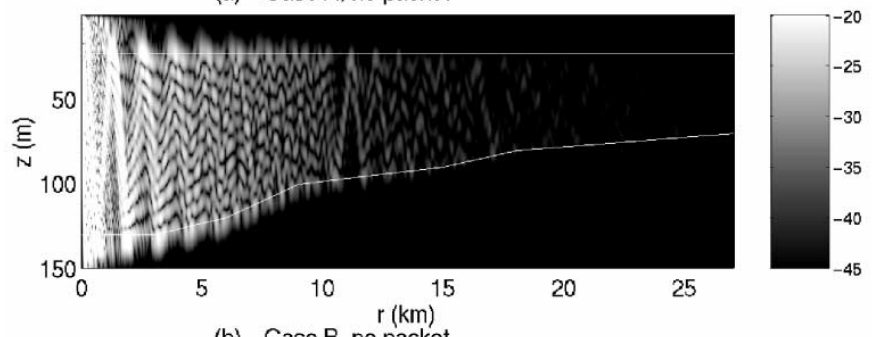

(b) Case B, no packet

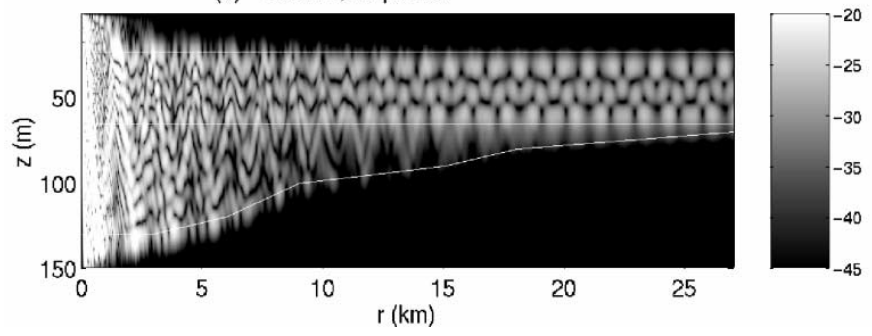

Fig. 6. Intensity is plotted for cases $\mathrm{A}$ and $\mathrm{B}$, each with no packet. Intensity is converted to decibels (re intensity at 1-m range from the source) after multiplication by the distance from the source to remove cylindrical spreading loss.

sity with cylindrical spreading removed (the "despread intensity") for case A (20-m source and no deep layer). The horizontal line shows the approximate interface depth between the upper and lower layers. Many modes are excited because the source is out of the main duct. The despread intensity level will remain unchanged in the absence of loss within the bottom, but it is seen to change in the figure because most of the modes excited by the source interact strongly with the bottom and, therefore, attenuate with range. Fig. 6(b) shows the case B despread intensity, which is much higher than the case A intensity and remains roughly constant in a range-averaged sense (averaged over a few kilometers) at ranges greater than about $5 \mathrm{~km}$ (i.e., at ranges where modes that interact with the bottom have been weakened and the signal is dominated by modes that are not bottom interacting). The white lines in Fig. 6(b) indicate the interfaces between the three layers. At ranges greater than $12 \mathrm{~km}$, case B shows interference of a few ducted modes, whereas case A shows continual decline of all mode amplitudes at all ranges.

Fig. 7(a) is comparable to Fig. 6(a), but shows the case $\mathrm{C}$ result with 50-m source. More low modes are excited than in case A, so total energy attenuation with range is reduced. Fig. 7(b) shows the associated case D result, with strong ducting and very little energy loss via bottom interaction.

Fig. 8(a) shows the despread intensity for case $E$ with the $100-\mathrm{m}$ source. There is a weak but steady decline of energy, with a few low-order modes present at the receiver. Fig. 8(b) shows the case F result, which has most of the energy trapped in bottom-interacting bottom-enhanced modes (probably mostly mode 4 , which is evident in the figure) and subsequently has lower intensity at the receiver than case $\mathrm{E}$.

Table II shows signal level at the receiver (27-km range) in each of the control cases (having no packet). The signal level at the receiver position is defined as the incoherent average over depth of acoustic field intensity (pressure amplitude squared) in the water $E=N^{-1} \sum_{n=1}^{N} a_{n}^{2}$ at 0.5 -m sampling interval, converted after averaging to units of transmission loss relative (a) Case C, no packet

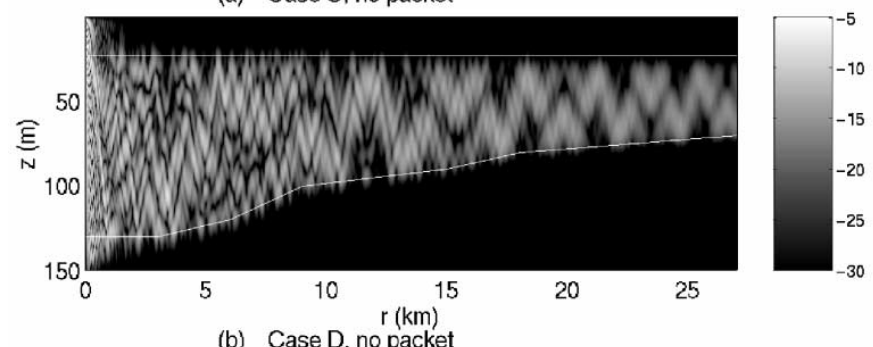

(b) Case D, no packet

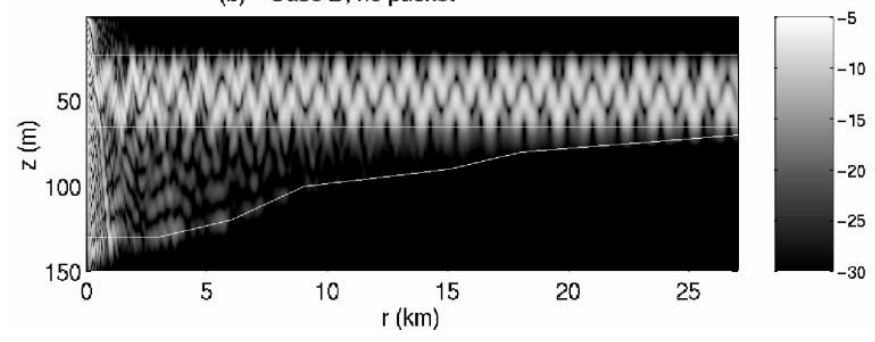

Fig. 7. Intensity with cylindrical spreading removed is plotted for cases $\mathrm{C}$ and D, each with no packet.

(a) Case E, no packet

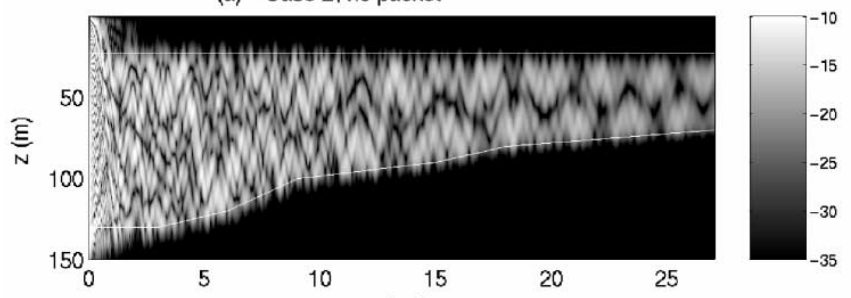

(b) Case F, no packet

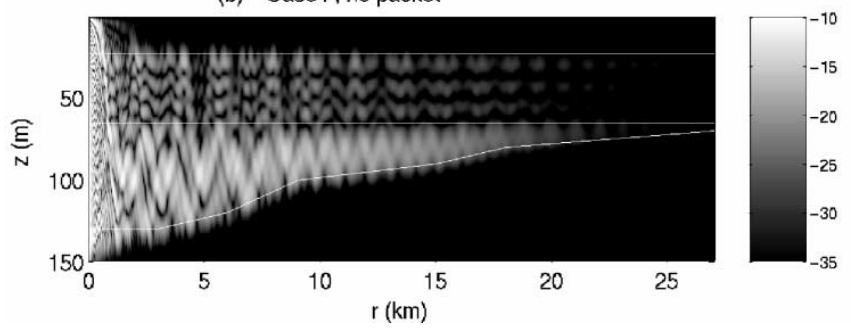

Fig. 8. Intensity with cylindrical spreading removed is plotted for cases $\mathrm{E}$ and $\mathrm{F}$, each with no packet.

TABLE II

DePTH-AVERAged ENERGy LEVELS E EXPRESSED IN TERMS OF TRANSMISSION LOSS AND GAIN EFFECT OF THE LAYER WITH NO WAVE PACKET PRESENT

\begin{tabular}{cccc}
\hline Source depth $(\mathrm{m})$ & $E$ without layer $(\mathrm{dB})$ & $E$ with layer $(\mathrm{dB})$ & Gain of layer $(\mathrm{dB})$ \\
\hline 20 & -106 & -87 & 19 \\
50 & -75 & -69 & 6 \\
100 & -77 & -95 & -18 \\
\hline
\end{tabular}

to pressure at 1-m range, as in previous work [2]. These signal levels include cylindrical spreading, so they do not correspond to the despread levels shown in Figs. 6-8. The weakest signal is for the near-surface source in the no-front system (case A). The strongest signal is for the mid-depth source with the front (with the deep layer; case D). The presence of the front has the least effect for the $50-\mathrm{m}$ source $(+6 \mathrm{~dB})$. The front causes $19 \mathrm{~dB}$ of gain for the upper source, but $18 \mathrm{~dB}$ of loss for the deep source. 

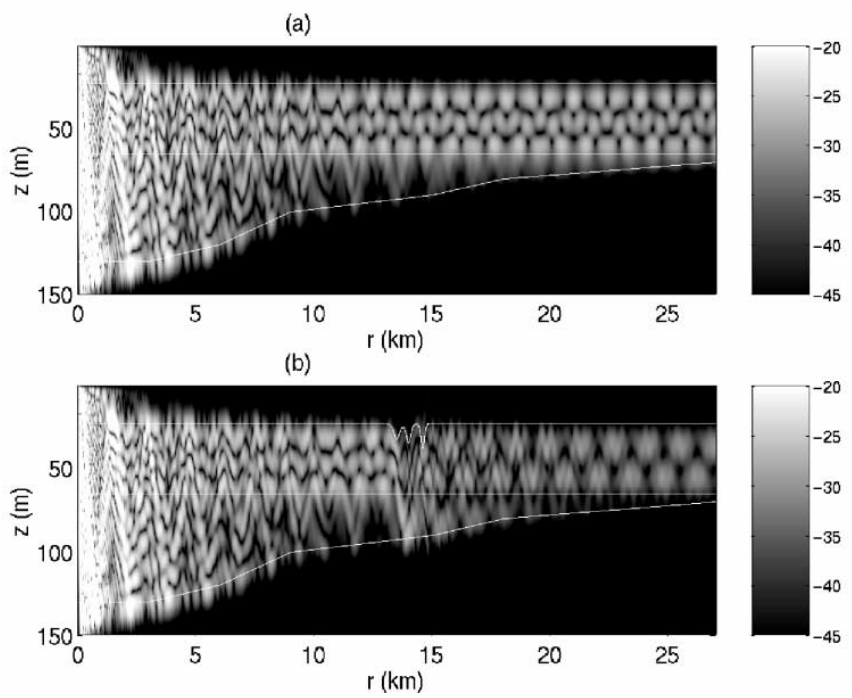

Fig. 9. (a) Acoustic field intensity, normalized so that cylindrical spreading is removed, is shown for case B with no packet. (b) The intensity, with spreading loss removed, for case B with a packet at the 13.5-km location (shown) is plotted. Decibel units are arbitrary but are directly comparable between the two panels. The approximate boundaries between the upper, middle, and deep layers are shown with white lines.

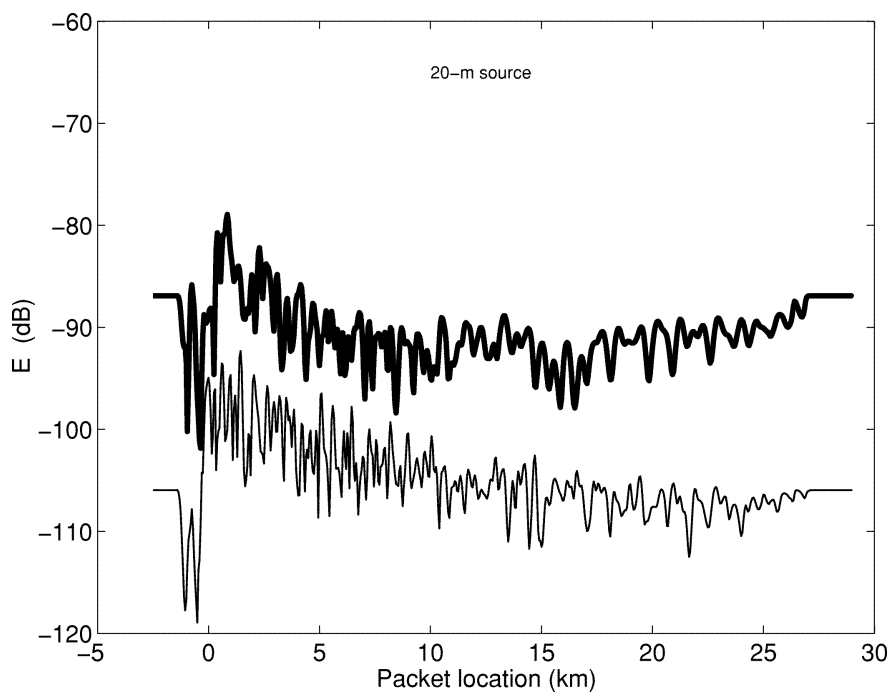

Fig. 10. Depth-averaged signal level $E$ (expressed in terms of transmission loss from a known source) at $27-\mathrm{km}$ range for the $20-\mathrm{m}$ depth source situations (cases A and B), plotted as a function of the location of a single solitary wave packet. The thin line shows case A (without the deep layer) and the thick line shows case B (with the deep layer). Packet position is measured as a function of distance from the source.

\section{B. Effect of Packets: 20-m Source Depth}

Next, we consider the effect of the model packet of steep nonlinear waves between the source and the receiver. Fig. 9 shows results from two case B runs, one without a packet and one with a packet at $13.5-\mathrm{km}$ range. (The packet range is given by the distance between the source and the wave trough closest to the source.) The lateral sound-speed gradients within the wave packet cause energy from the few modes that are present at the packet position (after $13 \mathrm{~km}$ of mode stripping) to couple into lossy bottom-interacting modes (or, equivalently, into high-angle bottom-penetrating rays). Therefore, less energy reaches the $27-\mathrm{km}$ site when the packet is present than when it is absent.

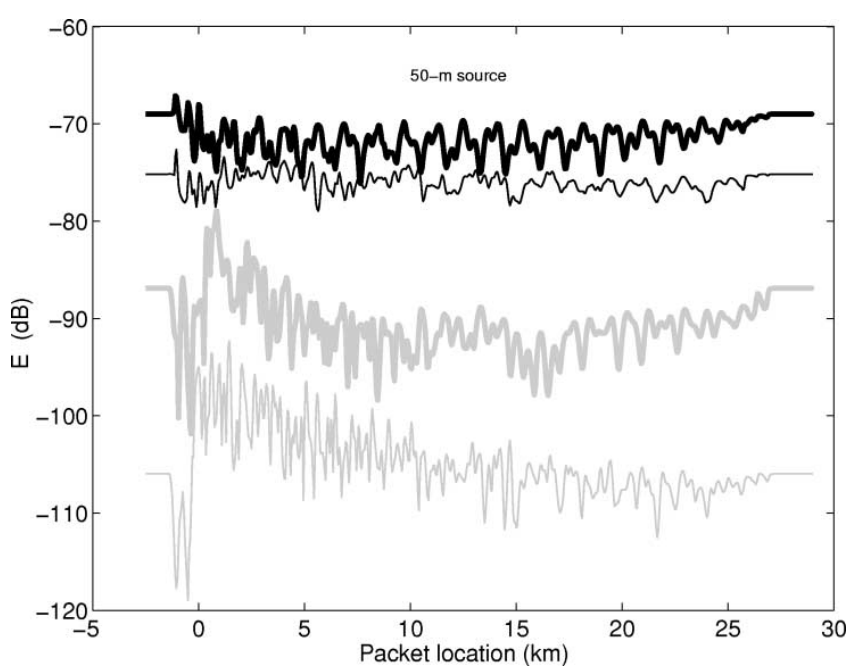

Fig. 11. Similar to Fig. 10, except that $E$ at 27-km range for a source at 50-m depth is shown. The thin line shows case $\mathrm{C}$ (without the deep layer) and the thick line shows case D (with the deep layer). The results for the 20-m source from the previous figure are shown dimly for comparison.

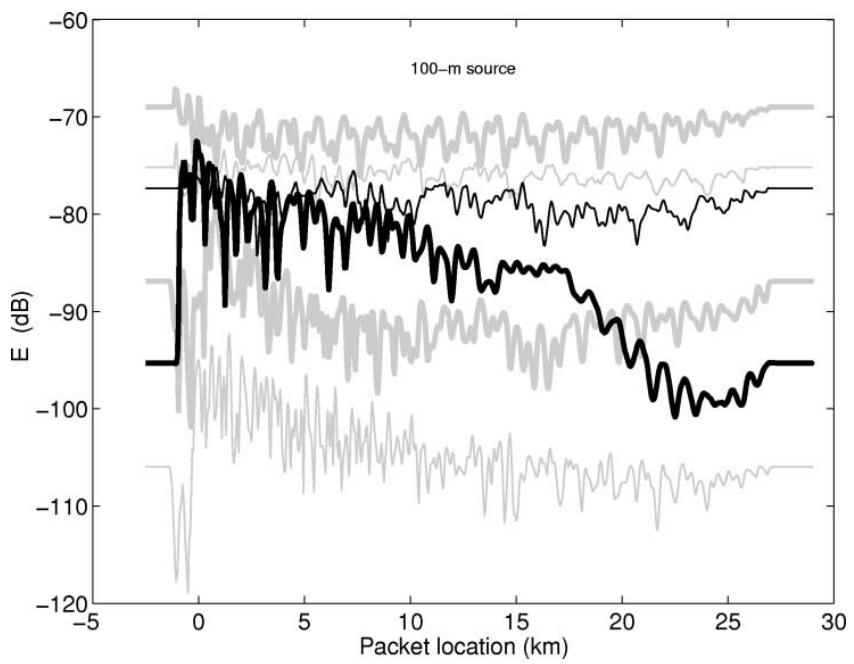

Fig. 12. Similar to Figs. 10 and 11, showing depth-averaged energy $E$ from a source at 100-m depth. The thin line shows case E, which does not have the deep layer, and the thick line shows case $\mathrm{F}$, which has the deep layer. The results of the previous two figures are included for comparison.

Fig. 10 shows the depth-integrated energy $E$ at 27-km range for packets at all ranges for cases A and B. This figure would represent a time series of received energy for a packet moving over the source and then continuing over the receiver. At a wave speed of $1 \mathrm{~m} / \mathrm{s}$, this would represent about $7.5 \mathrm{~h}$ of time. In this paper, we are interested in and will discuss signal fluctuations that occur over the longer packet displacement scales evident in the diagram (packet displacements of $3 \mathrm{~km}$ or greater), with the short-scale fluctuations averaged away. Low-pass filtered versions of $E$ curves from Figs. 10-12 are shown in Fig. 13 to facilitate discussion. The rapid fluctuation of $E$ over displacement scales of hundreds of meters was a topic of previous work [2] and is not discussed here, except to note that this behavior depends on modal phase interference. The Fig. 9(b) situation (case B, packet at $13.5 \mathrm{~km}$ ) is seen in Fig. 10 to have about $5 \mathrm{~dB}$ of loss relative to the no-wave [Fig. 9(a)] situation, which is seen at the extreme ends of the thick case B curve. 


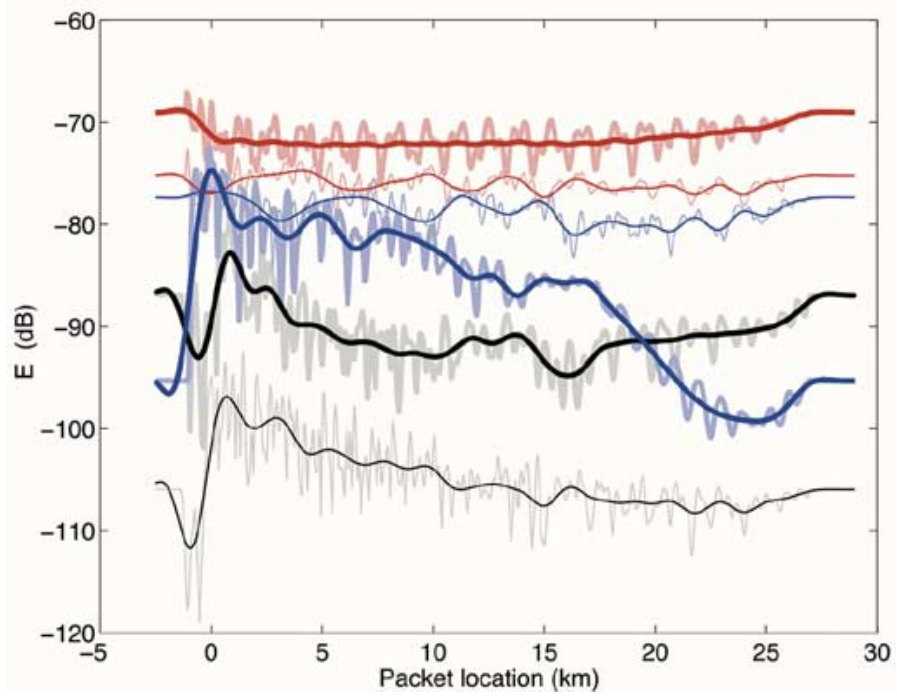

Fig. 13. Low-pass filtered versions of the results shown in the previous three figures are plotted. The original curves from those figures are shown lightly. The thin black line shows case A and the thick black line shows case B. The thin red line shows case $\mathrm{C}$ and the thick red line shows case $\mathrm{D}$. The thin blue line shows case $\mathrm{E}$ and the thick blue line shows case $\mathrm{F}$.

To summarize case A, the passage of the waves over the source causes loss because the source is moved fully into the upper layer and the efficiently propagating modes of the lower duct are poorly energized. As the packet moves fully past the source the effects of coupling from lossy to less-lossy modes is evident in the enhanced signal levels. $E$ is seen to increase by up to $15 \mathrm{~dB}$ for packets near the source. This signal gain decreases with increasing packet range until it is roughly zero at a packet range of $15 \mathrm{~km}$. Finally, some loss due to packets is noted for packet positions between 15 and $27 \mathrm{~km}$ because most acoustic energy encountering the packets is in efficiently propagating modes, so that coupling will tend to move energy into lossy modes.

In case $\mathrm{B}$, more of the source energy goes into modes that have less attenuation in the bottom, so the signal with no packet is higher than for case A. The packet effects are largely the same as for case A, except for the fact that the transition from preferential coupling into relatively lossless modes to preferential coupling into lossy modes occurs at $4-\mathrm{km}$ range rather than $15-\mathrm{km}$ range. This is because attenuation of lossy modes causes the lossless modes to dominate over the lossy modes (lossless modes contain a greater fraction of the total energy) closer to the source in case B than in case A.

\section{Effect of Packets: 50-m Source Depth}

Next, consider the effect of moving packets for the case of source depth at $50 \mathrm{~m}$ instead of $20 \mathrm{~m}$, with everything else left unchanged. Fig. 11 shows the results for cases C and D (without and with the deep layer). The packet merely introduces a low-amplitude nearly zero-mean fluctuation to the case $\mathrm{C}$ energy, corresponding to fluctuation between coupling of energy into high modes (giving loss) or into the lowest modes (giving gain) from the multitude of efficient (weakly attenuating) modes that are propagating efficiently in the lower layer duct. The lossy modes have lost all effect after only a few kilo- meters, leaving only the weakly attenuating low modes, so the effects of mode coupling are essentially range independent on the $2-\mathrm{km}$ or greater scale.

The effect with the layer (case D) is only slightly altered. The signal is a bit stronger because of the stronger ducting in the middle layer. There is slight signal loss from packet coupling because the energy becomes concentrated in a few weakly attenuating modes after only a few kilometers, so that the only effect that packet mode coupling can have is to move energy into modes with higher loss, causing signal reduction at the receiver.

\section{Effect of Packets: 100-m Source Depth}

Fig. 12 shows the effect of moving packets on received signal energy for a 100-m source depth, with all other parameters as in the other cases. The case $\mathrm{E}$ result is very much like the case $\mathrm{D}$ result, although with a shift in level. There is a slight loss due to mode coupling when the packet is at ranges of 15 or more kilometers, because in that situation the acoustic energy that encounters the packet is resident in efficiently propagating modes, so that coupling tends to induce signal loss. This effect is similar to the loss seen in case D, except in case E the low-loss modes do not dominate the total signal until 15-km range from the source is achieved, rather than 3-km range as in case D.

The case F result of Fig. 12 is similar in nature to the case A result: coupling causes signal gain when the packet is near the source, but causes loss when the signal is near the receiver (packet ranges 20-27 km). This situation of the source below the main duct and in the deep warm layer energizes mostly lossy higher modes. For this reason, coupling to low modes causes gain when the packet is $0-20 \mathrm{~km}$ from the source, a situation with the high modes carrying most of the energy adiabatically between the source and the packet (adiabatic meaning no coupling; there is attenuation). At 20-km range, the higher order modes have died away to the point where low-order modes begin to dominate, so coupling sends energy into the lossy high-order modes. Finally, as the packet moves past the receiver, the depthaveraged signal level rises to the no packet value of $-95 \mathrm{~dB}$.

\section{CONCLUSION}

Acoustic mode coupling induced by a packet of nonlinear internal waves in a coastal acoustic waveguide environment has been investigated with $400-\mathrm{Hz}$ computational propagation simulations. The ultimate effect on signal energy of coupling caused by packets interrupting otherwise adiabatic acoustic mode propagation over a $27-\mathrm{km}$ range is studied for two environments and compared. The first environment has a simple single thermocline configuration and the second adds a frontal feature akin to the Shalf Water-Slope Water Front south of New England [8], [13]. The simulated water column is horizontally layered except for the wave packet in all cases. The effect of low-amplitude low-slope internal waves would be to cause fluctuations of a fraction of a decibel root mean square (rms) [2], an effect not expected to alter the applicability of the results presented here.

The effects of coupling on received depth-averaged intensity are markedly different for the two situations and the effects are markedly different for three different source depths. Results range from $20-\mathrm{dB}$ gain for a packet near a 100-m depth acoustic 


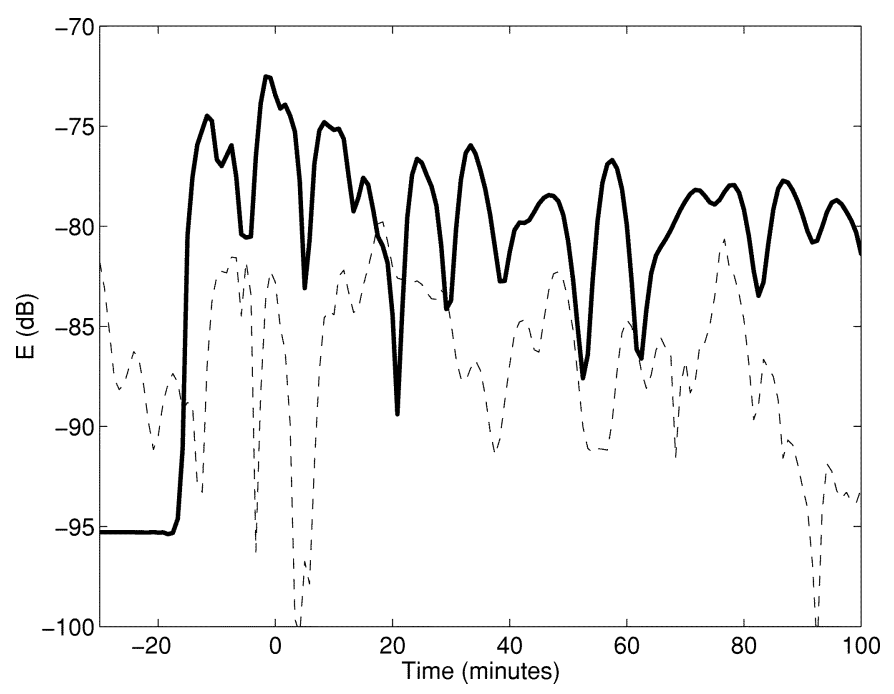

Fig. 14. Depth-averaged energy $E$ for a source at 100-m depth for two cases: a single packet (thick line) and six packets (dashed line) between the source and receiver, both with the front (deep warm layer) present. The single-packet result (thick line) is case $\mathrm{F}$ and is repeated from Fig. 12. The packet location variable has been converted to time using a packet speed of $1 \mathrm{~m} / \mathrm{s}$. The packets remain equally spaced in the simulation (all packets are given equal speed).

source in the frontal situation (case F) to 8-dB loss for a packet $15 \mathrm{~km}$ from the source in the frontal case with 20 -m source depth (case B).

With other parameters held equal, the most pronounced effect of the front on packet gain/loss occurs with the source at 100-m depth. With no front, the packet causes a few decibels of signal loss. However, with the front present, the packet causes $20 \mathrm{~dB}$ of gain when it is within $10 \mathrm{~km}$ of the source (Fig. 12). Note also that the presence of the front weakens the signal when packet-induced coupling is not occurring, so that the gain caused by packet coupling merely brings the signal up to the level it would have (regardless of whether packets cause coupling) in the absence of the front.

The approximately $20 \mathrm{~dB}$ of average gain caused by the single near-source packet near the 100-m source case in the presence of the front (Fig. 12, case F) is measured with respect to the very low levels of the packet-free situation and also corresponds to the situation of no other packets between the source and receiver. Experiments such as Shelfbreak PRIMER [6], [14] and SWARM [15], [16] indicate that multiple packets are likely to be present in a $27-\mathrm{km}$ upslope propagation path. This means that the 20-dB gain seen in Fig. 12 as a packet passes over the source in an otherwise wave-free domain is not likely to be seen in upslope propagation experiments such as those. Instead, observations from those experiments might be better compared with a simulation having multiple packets moving between the source and receiver. Fig. 14 shows the result when five additional packets are added to the case F situation, each moving in the same (idealized) manner as the original packet. The case F result of Fig. 12 is also shown in Fig. 14. The highest signal level of the multiple-packet simulation is about $7 \mathrm{~dB}$ lower than the single-packet simulation, as is the average level. It is more appropriate to compare the levels and temporal behavior of $E$ observed in the cited experiments to the multiple-packet simulation results than to the single-packet simulation results con- tained in the bulk of this paper. The single-packet simulations are intended to show the physical effects more clearly.

All of the signal gain or loss effects caused by packets that are shown in this paper can be deduced in a straightforward way from the basic principles of mode propagation. Said differently, they follow directly from those principles. Three process are at work in each of the six cases studied here:

1) modal excitation by localized sources at specific depths;

2) differential attenuation of various modes (mode stripping) during adiabatic mode propagation;

3) redistribution of energy from excited to depleted modes (a diffusion-like transfer process) by the coupling process, a process that acts approximately like six discrete coupling events for our three-wave packets.

Expanding on (3), previous work has shown that mode coupling activity in solitary-type internal waves is concentrated where the thermocline is steeply sloped, so that there are two coupling regions for a symmetric wave [1]. The intent of this study is to illustrate the varying effects, in different environments, of mode coupling induced by packets of steep internal waves. Variations of the interactions between the three processes (mode excitation, mode stripping, and coupling transfer) can give rise to many behaviors. Large variability in energy level is evident in the two example environments considered here, which were chosen to be representative of the Mid-Atlantic Bight and possibly other coastal regions. Other complicated behavior is likely to occur for coupled-mode propagation in other environments.

\section{ACKNOWLEDGMENT}

J. Preisig, G. Frisk, and J. Lynch contributed computational resources and useful comments.

\section{REFERENCES}

[1] J. C. Preisig and T. F. Duda, "Coupled acoustic mode propagation through continental-shelf internal solitary waves," IEEE J. Oceanic Eng., vol. 22, pp. 256-269, Apr. 1997.

[2] T. F. Duda and J. C. Preisig, "A modeling study of acoustic propagation through moving shallow-water solitary wave packets," IEEE J. Oceanic Eng., vol. 24, pp. 16-32, Jan. 1999.

[3] D. Rouseff, A. Turgut, S. N. Wolf, S. Finette, M. H. Orr, B. H. Pasewark, J. R. Apel, M. Badiey, C.-S. Chiu, R. H. Headrick, J. F. Lynch, J. N. Kemp, A. E. Newhall, K. von der Heydt, and D. Tielbuerger, "Coherence of acoustic modes propagating through shallow water internal waves," J. Acoust. Soc. Amer., vol. 111, pp. 1655-1666, 2002.

[4] D. Tielburger, S. Finette, and S. Wolf, "Acoustic propagation through the internal wave field in a shallow water waveguide," J. Acoust. Soc. Amer., vol. 101, pp. 789-808, 1997.

[5] D. M. Milder, "Ray and wave invariants for SOFAR channel propagation," J. Acoust. Soc. Amer, vol. 46, pp. 1259-1263, 1969.

[6] J. F. Lynch, A. E. Newhall, B. Sperry, G. Gawarkiewicz, A. Fredricks, P. Tyack, C.-S. Chiu, and P. Abbot, "Spatial and temporal variations in acoustic propagation characteristics at the New England shelfbreak front," IEEE J. Oceanic Eng., vol. 28, pp. 129-150, Jan. 2003.

[7] G. C. Chang and T. D. Dickey, "Optical and physical variability on timescales from minutes to the seasonal cycle on the New England shelf: July 1996-June 1997," J. Geophys. Res., vol. 106, pp. 9435-9453, 2001.

[8] C. R. Rehmann and T. F. Duda, "Diapycnal diffusivity inferred from scalar microstructure measurements near the New England shelf/slope front," J. Phys. Oceanogr., vol. 30, pp. 1354-1371, 2000.

[9] T. F. Duda and C. R. Rehmann, "Systematic microstructure variability in double-diffusively stable coastal waters of nonuniform density gradient," J. Geophys Res., vol. 106, p. 3145, 2002. doi:10.1029/2001JC000844. 
[10] T. F. Duda, "Relative influences of various environmental factors on 50-1000 Hz sound propagation in shelf and slope areas," in Impact of Littoral Environmental Variability on Acoustic Predictions and Sonar Performance, N. G. Pace and F. B. Jensen, Eds. Dordrecht, The Netherlands: Kluwer, 2002, pp. 393-400.

[11] M. D. Collins and E. K. Westwood, "A higher-order energy-conserving parabolic equation for range-dependent ocean depth, sound speed, and density," J. Acoust. Soc. Amer., vol. 89, pp. 1068-1075, 1991.

[12] A. K. Rogers, T. Yamamoto, and W. Carey, "Experimental investigation of sediment effect on acoustic wave propagation in shallow water," $J$. Acoust. Soc. Amer, vol. 93, pp. 1747-1761, 1993.

[13] A. D. Voorhis, D. C. Webb, and R. C. Millard, "Current structure and mixing in the shelf/slope water front south of New England," J. Geophys. Res., vol. 81, pp. 3695-3708, 1976.

[14] J. A. Colosi, R. C. Beardsley, G. Gawarkiewicz, J. F. Lynch, C.-S. Chiu, and A. Scotti, "Observations of nonlinear internal waves on the outer New England continental shelf during the summer shelfbreak PRIMER study," J. Geophys. Res., vol. 106, pp. 9587-9601, 2001.

[15] R. H. Headrick, J. F. Lynch, J. N. Kemp, A. E. Newhall, K. von der Heydt, J. Apel, M. Badiey, C.-S. Chiu, S. Finette, M. Orr, B. Pasewark, A. Turgut, S. Wolf, and D. Tielbuerger, "Modeling mode arrivals in the 1995 SWARM experiment acoustic transmissions," J. Acoust. Soc. Amer., vol. 107, pp. 221-236, 2000.
[16] J. R. Apel, M. Badiey, C.-S. Chiu, S. Finette, R. Headrick, J. Kemp, J. F. Lynch, A. Newhall, M. H. Orr, B. H. Pasewark, D. Tielbuerger, A. Turgut, K. von der Heydt, and S. Wolf, "An overview of the 1995 SWARM shallow water internal wave acoustic scattering experiment," IEEE J. Oceanic Eng., vol. 22, pp. 465-500, July 1997.

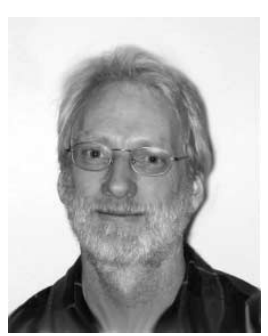

Timothy F. Duda received the B.A. degree in physics from Pomona College, Claremont, CA, in 1979 and the Ph.D. degree in oceanography from the Scripps Institution of Oceanography, University of California, San Diego, in 1986.

He has been a Scientist at the Woods Hole Oceanographic Institution, Woods Hole, MA, since 1991. Prior to that, he held positions at the University of California, Santa Cruz. His three primary fields of study are ocean acoustic propagation, ocean internal gravity waves, and ocean mixing processes. He has modeled and analyzed fluctuations of ocean acoustic transmissions, has made electromagnetic-type internal-wave measurements, and performed in situ measurements of ocean microstructure.

Dr. Duda is a Member of the American Meteorological Society, the American Geophysical Union, and the Acoustical Society of America. 